zumal die Beiträge nicht immer aufeinander abgestimmt sind und somit zahlreiche Wiederholungen enthalten. Überhaupt stellt sich die Frage, welche Wichtigkeit die reine Faktenvermittlung heute noch haben kann angesichts des glücklichen Umstandes, daß Osteuropa weitgehend für Menschen und Massenmedien frei zugänglich geworden ist. Vor diesem Hintergrund ist ein gewisses Theoriedefizit nicht nur in einzelnen Beiträgen, sondern auch in der Gesamtkonzeption - wohl der Tagung - festzustellen. Es hätte sich angeboten, gegebenenfalls zu Lasten der (nur) faktenorientierten Beiträge einen demokratietheoretischen Beitrag und einen allgemeinen Beitrag zu Transformationstheorien unter vergleichender Einbeziehung autoritärer oder ehemals autoritärer Präsidialsysteme aufzunehmen. Zuweilen, auch dies betrifft die nicht immer gebotene Theorietiefe einzelner Beiträge, fällt ein gewisser Hang zu Selbstzitaten auf, wodurch die teilweise geringe Berücksichtigung der doch sehr umfangreichen einschlägigen Literatur in Ost und West nicht immer ausgeglichen werden kann.

Insgesamt handelt es sich um ein Buch, das den Blick schärft für Probleme von Selbstfindungsprozessen, nicht nur der Transformationsstaaten im Osten, sondern auch der Osteuropawissenschaft im Westen.

Oesten Baller

\title{
International Organizations and the Law of the Sea. Documentary Yearbook 1994
}

Issued by the Netherlands Institute for the Law of the Sea (NILOS), Vol. 10, 1994

Graham and Trotman / Martinus Nijhoff, London, 1996, 894 pp., $£ 249.00$

Alfred Soons und Barbara Kwiatkowska vom Niederländischen Seerechtsinstitut geben seit zehn Jahren die Dokumentation über seerechtliche Aktivitäten Internationaler Organisationen heraus. Der vorliegende 10. Band enthält die Ergebnisse des wichtigen Jahres 1994, in dem das neue UN-Seerechtsübereinkommen in Kraft trat.

Wie in früheren Bänden werden wichtige Dokumente im englischen Originaltext reproduziert, andere - aus Platzgründen - lediglich mit Titel und Fundstelle aufgelistet, insgesamt 258 Dokumente, davon 93 in voller Textlänge abgedruckt. Angesichts der Vielfalt, des Umfangs und vielleicht auch der unterschiedlichen Bedeutung der Dokumente ist diese selektive Vorgehensweise unumgänglich.

Eine wichtige Einschränkung liegt in der Ausrichtung auf die Vereinten Nationen und ihre Organisationen, d.h. seerechtliche Dokumente anderer internationaler Organisationen wie z.B. der Europäischen Union, der Helsinki-Kommission (für die Ostsee) oder der IOMAC (für den Indischen Ozean) findet man in dieser Dokumentation nicht.

Abgesehen von diesen notwendigen Einschränkungen bleiben für den Leser kaum Wünsche offen, denn das gesamte seerechtliche und meerespolitisch/wirtschaftliche Wirken der Familie der UN-Organisationen findet sich in selbständigen Kapiteln unterteilt nach UN- 
Generalversammlung, den unterschiedlichen Seerechts- und Fischereigremien, ECOSOC, ECE, ESCAP, UNEP und UNCTAD gefolgt von den eigenständigen Sonderorganisationen FAO, IAEA, ILO, IMO, UNESCO und WHO.

Als besonders wertvoll erweist sich im Meer der UN-Aktivitäten der jährliche Seerechtsbericht des Generalsekretärs (S. 17-75) und die ebenfalls jährlich wiederkehrende Seerechtsresolution (S. 223 f.), die dem eiligen Leser den Überblick über alle wesentlichen Entwicklungen geben. Herausragende seerechtliche Bedeutung haben z.B. die informellen Konsultationen zum Seerechtsübereinkommen, die Arbeiten der Vorbereitungskommission und die wichtige Resolution 48/263 zum Implementierungs- (Durchführungs-) Übereinkommen, die den Weg zum Inkrafttreten des Seerechtsübereinkommens im Jahre 1994 freimachten. Ein anderer Meilenstein, der in diesem Band dokumentiert wird, sind die Verhandlungen zur Konvention über wandernde Fischarten, die 1995 erfolgreich abgeschlossen wurden.

Die Vielfalt der Einzelthemen, die sich hier nur andeuten läßt, reicht von der Antarktis über die Seegrenzverhältnisse Guatemalas bis hin zu Arbeitsgruppenberichten der IMO über die Novellierung der Londoner Dumping-Konvention. Das Material umfaßt also globale Konventionen und Konferenzen, regionale Entwicklungen und nationale Tatbestände in Gestalt der zahlreichen schriftlichen Erklärungen der Staaten.

Ein leider etwas knapp gehaltenes Stichwortverzeichnis und die Adressen für die Bezugsquellen der nicht abgedruckten Dokumente dienen der Benutzerfreundlichkeit. Das Werk ist angesichts der wachsenden Globalisierung des internationalen Seerechts und der starken Rolle der Vereinten Nationen und ihrer Sonderorganisationen für alle Wissenschaftler und Praktiker in Regierung und Verwaltung unentbehrlich, die sich mit dem Seerecht, aber auch mit Meeresnutzungen beschäftigen.

Uwe Jenisch

\section{J. Ashley Roach / Robert W. Smith}

United States Responses to Excessive Maritime Claims, Second Edition

Martinus Nijhoff Publishers, The Hague, 1996, 676 pp.

(Publications on Ocean Development, Vol. 27)

Die beiden im US State Department tätigen Autoren Roach und Smith haben mit diesem seerechtspolitischen Werk in kurzer Folge auf die 1. Auflage unter dem damaligen Titel "Excessive Maritime Claims" (Besprechung in VRÜ 28 [1995], S. 566) erneut eine Bilanz über die aktuelle Staatenpraxis zur Ausdehnung der hoheitlichen Meereszonen vorgelegt.

Die fast doppelt so umfangreiche 2. Auflage war notwendig geworden, weil das UN-Seerechtsübereinkommen von 1982 am 16. November 1994 in Kraft trat. Bisher sind rund 110 Staaten dem Übereinkommen beigetreten. Die 2. Auflage versteht sich auch als ein Beitrag 